\title{
PANCREATIC STEATOSIS AND ITS ASSOCIATION WITH NONALCOHOLIC FATTY LIVER DISEASE EVALUATED BY ULTRASONOGRAPHY
}

Luis Jesuino de Oliveira Andrade*, Laryssa Rocha Guimarães**, Raissa Brito Fernandes Cadete**, Hudson Sá Sodré***, Paulo Roberto Santana de Melo****, Emmanuel Conrado de Souza ${ }^{* * * * *}$

Corresponding author: Luis Jesuino de Oliveira Andrade luis_jesuino@yahoo.com.br

* Professor at the State University of Santa Cruz, Faculty of Medicine (UESC) - Bahia

** Santa Casa de Itabuna - Bahia

*** Medicine Undergraduate student at State University of Santa Cruz (UESC) - Bahia

**** Professor at the State University of Santa Cruz Faculty of Medicine (UESC) - Bahia

***** State University of Santa Cruz Faculty of Medicine (UESC) - Bahia

\begin{abstract}
Introduction: Pancreatic steatosis (PS) is the fatty replacement of pancreatic parenchyma, being often associated with obesity and aging. Similar to this condition in pancreas, any excessive lipidic deposition in the liver tissue are referred to as nonalcoholic fatty liver disease (NAFLD). Objective: The objective of this study was to explore associations between PS and the NAFLD grade, as evaluated by ultrasonography. Methods: This was a cross-sectional study. Diagnosis of the PS and NAFLD was based on ultrasound findings, and 576 ultrasonography exams were evaluated. The chi-square test was used for evaluation of the relationship between PS and NAFLD. Results: A total of 576 individuals were included, 217 (or $37.7 \%$ ) were male and 359 (or 62.3\%) were female. The median age of the patients was 47.04 (11.8) years-old (mean \pm standard error). Among them, 243 were diagnosed with PS and 245 were diagnosed with NAFLD according to the trans-abdominal ultrasound findings. There was an association between male sex and PS (P 0.012), and between female sex and NAFLD (P 0.0001). As compared to the normal group, the PS and NAFLD group was characterized by a significantly higher median age. There was also a statistically significant association between the degree of PS and the degree of NAFLD, with the following characteristics: mild PS and mild NAFLD (P 0.0001), moderate PS and moderate and severe NAFLD ( $P$ 0.0001) and between severe PS and moderate and severe NAFLD ( $P$ 0.0001). Conclusion: Our findings demonstrated that fat accumulation in pancreas is significantly correlated to NAFLD. It was also shown that the ultrasound is very resourceful when it comes evaluation of fat grade. Keywords: Pancreatic steatosis; Liver steatosis; Nonalcoholic fatty pancreas disease; Nonalcoholic fatty liver disease; Ultrasonography.
\end{abstract}




\section{INTRODUCTION}

The fatty infiltration of the pancreas was first described in 1993 by Ogilvie that reported 17\% pancreatic fat storage for obese cadavers as compared with $9 \%$ for lean ones. Pancreatic steatosis (PS) is the best description for fat accumulation in the pancreatic gland without fat replacement. (1) However, several synonyms have been used for describing the accumulation of fat in pancreas, such as non-alcoholic fatty pancreatic disease, pancreatic lipomatosis, fatty replacement, fatty infiltration, fatty pancreas, and lipomatous pseudo-hypertrophy. ${ }^{(2)}$

The PS is a fatty replacement of pancreatic parenchyma, being often associated with obesity and aging. ${ }^{(3)}$ The PS is generally a diffuse process occurring uniformly, but may be unevenly distributed in the pancreas and can be confined to region of the pancreas (focal fatty infiltration), being classified as: type 1a: preferential fatty replacement of head; type ib: preferential fatty replacement of head, neck and body; type 2a: preferential fatty replacement of head and uncinate process; type $2 \mathrm{~b}$ : fatty replacement of most of pancreas regions except the peribiliary region. (4) Focal PS is a disorder without clinical significance. However, this condition may simulate a mass-like lesion.

It is speculated that nonalcoholic fatty liver disease (NAFLD) begets PS. However, it has been demonstrated that the PS begets the development of NAFLD. ${ }^{(5)}$ Similar to the condition in pancreas, excessive lipid deposition in liver is referred to as NAFLD. The NAFLD pathogenesis is still largely unknown, and the key factor in the pathophysiology of NAFLD is the insulin resistance that determines lipid accumulation in the hepatocytes. ${ }^{(6)}$

Several diagnostic techniques by imaging have been applied in quantifying pancreatic fat such as transabdominal ultrasound, endoscopic ultrasonography, computed tomography and magnetic resonance imaging. ${ }^{(7)}$ However, the most largely used technique is transabdominal ultrasonography. ${ }^{(8)}$
The aim of this study was to investigate the prevalence of PS and its association with NAFLD grade, as evaluated by ultrasonography.

\section{METHODS}

We reviewed retrospectively exams of patients that were submitted to an abdominal ultrasound between November 2013 and April 2015. Since this is a retrospective study without consequences for the patients involved, it was not signed informed consent by patients or guardians, and the study did not need to be submitted to an institutional review board.

The study evaluated only sonographic aspects, not assessing and correlating other information on the study population, such as body mass index, glycemic status or other metabolic aspects.

Pancreas and liver ultrasonography were performed simultaneously by a single experienced imaging specialist, to minimize biases by different specialists, with high resolution ultrasonography (Toshiba Xario XG) using a 3.5 MHz convex probe. A complete ultrasound scan was performed with the subjects in supine position. The pancreas region was traced covering the area from the pancreatic head, body and tail to the level of the superior mesenteric artery. The liver was examined from sub- and intercostally in the decubitus position as well as in modified slightly oblique positions with the right arm above the head and the right leg stretched during all respiration cycles to identify the best approach and to avoid artifacts caused by the thorax.

The PS was diagnosed when there was an increase in echogenicity of the pancreas compared with the kidney. As the pancreas echogenicity could not be compared directly with the kidney in the same window, the examiner compared the difference between pancreatic echogenicity and the renal echogenicity in different window, and between hepatic and pancreatic echogenicity, to obtain an objective pancreato-renal echo contrast (Figure 1). The PS can be classified as diffuse or as focal fatty infiltration, this latter was classified as: type 
1a: preferential fatty replacement of head; type $1 \mathrm{~b}$ : preferential fatty replacement of head, neck and body; type 2a: preferential fatty replacement of head and uncinate process; type $2 b$ : fatty replacement of most of pancreas except peribiliary region. (4) However, we subdivided the PS into mild (grade I), moderate (grade II), and severe (grade III), according with Lee et al criteria. The four echogenicity grades of PS: Non-fatty pancreas, pancreatic echogenicity is equal to renal cortical echogenicity; Grade I: Mild fatty pancreas, pancreatic echogenicity is definitely lower than retroperitoneal fat; Grade II: Moderate fatty pancreas, pancreatic echogenicity is slightly lower than retroperitoneal fat; and Grade III: Severe fatty pancreas, pancreatic echogenicity is equal to retroperitoneal fat. ${ }^{(8)}$

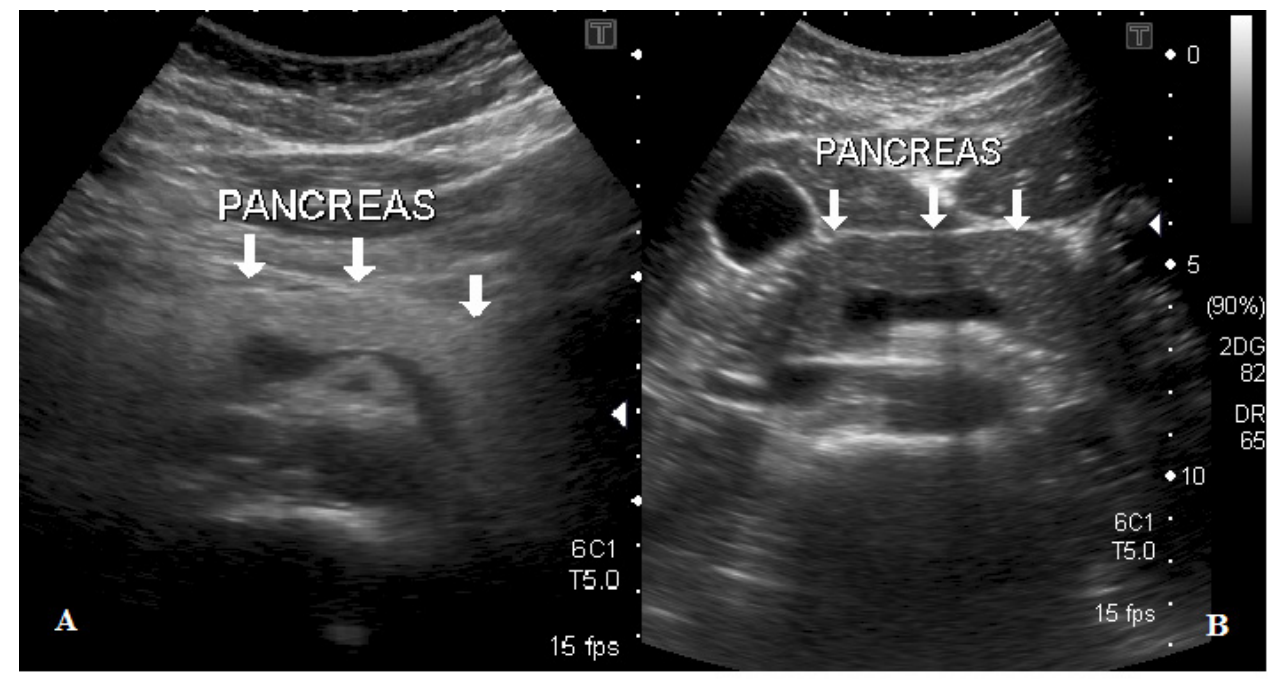

Figure 1. A - Difuse steatosis pancreatic

B. Normal pancreas

The NAFLD diagnostic criteria included characteristic echo patterns of hepato-renal echo contrast, bright liver, deep attenuation, and vascular blurring (Figure 2). The Grading of NAFLD was classified as: grade I (mild): increased hepatic echogenicity with visible periportal and

diaphragmatic echogenicity; grade II (moderate): increased hepatic echogenicity with imperceptible periportal echogenicity, without obscuration of diaphragm; grade III (severe): increased hepatic echogenicity with imperceptible periportal echogenicity and obscuration of diaphragm. ${ }^{(9)}$

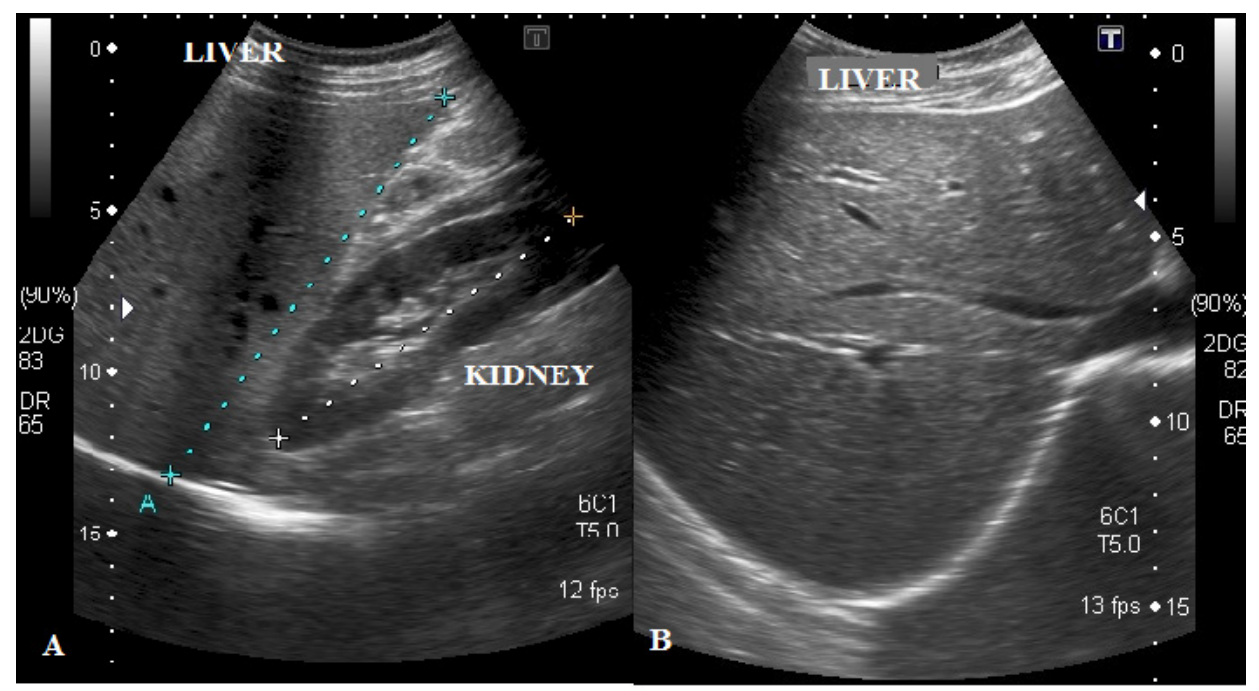

Figure 2. A - Steatosis liver

B - Normal liver 


\section{STATISTICAL ANALYSES}

All normally distributed continuous variables were expressed as means $\pm S D$. The chi-square test was used to analyze for evaluation of the relationship between PS and NAFLD. A P-value less than 0.05 was considered statistically significant.

\section{RESULTS}

A total of 576 ultrasonography exams were evaluated. Among them, 243 were diagnosed with PS according to the trans-abdominal ultrasonographic findings; 217 (37.7\%) were male and 359 (62.3\%) were female. Two hundred forty-five were diagnosed with NAFLD according to the trans-abdominal ultrasonographic findings; 120 (20.8\%) were male and $123(21.3 \%)$ were female. The 576 patients' mean age was 47.04 (11.8) yearsold (mean \pm standard error). The general characteristics, PS, NAFLD, and normal groups are shown in Table 1.

Table 1 - General characteristics, PS, NAFLD, and normal groups

\begin{tabular}{ccccc}
\hline VARIABLE & ALL & PS & NAFLD & NORMAL \\
\hline N & 576 & 243 & 245 & 261 \\
GENERAL CHARACTERISTICS & & & & \\
MALE & 217 & 106 & 120 & 81 \\
$(\%)$ & $(37.7)$ & $(18.4 \%)$ & $(20.8 \%)$ & $(14.0 \%)$ \\
FEMALE & 359 & 137 & 124 & 180 \\
$(\%)$ & $(62.3 \%)$ & $(23.7 \%)$ & $(21.5 \%)$ & $(31.2 \%)$ \\
AGE & $47.04(15.92)$ & 65.07 & 69.50 & 33.50 \\
(MEAN FFL SD YEARS) & & $(8.27)$ & $(10.14)$ & $(14.05)$ \\
\hline
\end{tabular}

There was an association between male sex and PS ( $P$ 0.012), and between female sex and NAFLD $(P$ 0.0001). As compared to the normal group, the PS and NAFLD group was characterized by a significantly higher median age. There was also a statistically significant association between the degree of PS and the degree of NAFLD, with the characteristics following: mild PS and mild NAFLD ( $P$ 0.0001), moderate PS and moderate and severe NAFLD $(P 0.0001)$ and between severe PS and moderate and severe NAFLD ( $P$ 0.0001) (Table 2). 
Table 2 - PS degree * NAFLD degree Cross tabulation

\begin{tabular}{|c|c|c|c|c|c|c|}
\hline \multicolumn{7}{|c|}{ NAFLD DEGREE } \\
\hline \multirow{6}{*}{ PS degree } & & $\begin{array}{l}\text { Normal } \\
\text { (n) }\end{array}$ & $\begin{array}{l}\text { Mild fatty } \\
\text { (n) }\end{array}$ & $\begin{array}{c}\text { Moderate } \\
\text { fatty } \\
\text { (n) }\end{array}$ & $\begin{array}{l}\text { Severe fatty } \\
\text { (n) }\end{array}$ & $\begin{array}{c}\text { Total } \\
\text { (n) }\end{array}$ \\
\hline & $\begin{array}{l}\text { Normal } \\
\text { (n) }\end{array}$ & 261 & 38 & 25 & 9 & 333 \\
\hline & $\begin{array}{l}\text { Mild fatty } \\
\text { (n) }\end{array}$ & 50 & 43 & 26 & 9 & 128 \\
\hline & $\begin{array}{l}\text { Moderate fatty } \\
\text { (n) }\end{array}$ & 12 & 18 & 29 & 16 & 75 \\
\hline & $\begin{array}{l}\text { Severe fatty } \\
\text { (n) }\end{array}$ & 8 & 8 & 14 & 10 & 40 \\
\hline & $\begin{array}{l}\text { Total } \\
\text { (n) }\end{array}$ & 331 & 107 & 94 & 44 & 576 \\
\hline
\end{tabular}

\section{DISCUSSION}

In this study, using ultrasonography that allows the non-invasive evaluation of PS and NAFLD was demonstrated that PS is common in patients with NAFLD, and the fatty infiltration of pancreas positively correlates with fatty infiltration of liver ultrasound-determined steatosis grade. This finding is compatible with those of an autopsy report ${ }^{(10)}$ and with others of several studies involving the use of ultrasonography, ${ }^{(8)}$ endoscopic ultrasonography, ${ }^{(11)}$ computed tomography, ${ }^{(12)}$ and magnetic resonance imaging. ${ }^{(13)}$

Some researchers believe that PS or fat deposition in pancreatic cells does not exist in humans, ${ }^{(14)}$ but studies with pathological observations on the human pancreas, showed that fat deposition in the human pancreas occur mainly in interlobular septa. (8) The ultrasonography has been used as a tool for evaluation of fat infiltration in the pancreas and liver. The occurrence of fatty pancreas and fatty liver on ultrasonography was very high in our study.

PS can be justified owing to the coexistence with NAFLD. Beyond of fat infiltration in the liver, other factors associated with fat infiltration in the pancreas include age, increased body mass index, metabolic syndrome, and alcohol use, ${ }^{(15)}$ that was not assessed in our study.

There is well documented association between advanced age and PS. ${ }^{(16)}$ A report found that fat deposition was significantly increased in pancreas islet cells of individuals aged over 60 years. ${ }^{(17)}$ The evaluation with endoscopic ultrasonography and autopsy studies are consistent with this association. (5) However, a prospective study with endoscopic ultrasonography did not show this association. ${ }^{(11)}$ Similarly, the prevalence of NAFLD in the general population increases with age, and fatty liver is more prevalent in men than women up to the age of 60 years. Beyond menopause, the prevalence of fatty liver rises sharply in women and exceeds that observed in their male counterparts. ${ }^{(18)}$ In the present study, we showed that advanced age was positively associated with PS and NAFLD, and is compatible with the results of previous studies, possibly due to the age-related decrease in pancreatic parenchymal volume and increased pancreatic fat content, as well as fatty replacement of damaged tissue of liver in the older subjects.

The PS and NAFLD are an increasing problem due to the increasing incidence of obesity. The presence of fatty pancreas and fatty liver represents 
a meaningful manifestation of metabolic syndrome together with obesity. ${ }^{(5)}$ The pancreatic fat replacement with acinar cell death and pancreatic fat infiltration due to obesity contribute to PS. ${ }^{(2)}$ Likewise, obesity is the most significant single risk factor for the development of fatty liver, both in children and in adults. ${ }^{\left({ }^{19}\right)}$ The association between PS and NAFLD with obesity was not assessed in our study.

Another important risk factor for pancreatic fat infiltration and liver fat infiltration is alcohol ingestion. Fat infiltration within pancreatic acinar cells was observed by examination in patients consuming more than $30 \mathrm{gr} /$ day of ethanol. Ultrastructural changes within the cells are described on those without clinical pancreatitis, changes which are similar to those seen in the hepatocytes of alcoholics. ${ }^{(17)}$ Our study, nevertheless, did not include individuals who consume alcoholic beverages.

The definition of metabolic syndrome remains a matter of debate, however, obesity, hyperglycemia, dyslipidemia and hypertension have been constant syndrome components and the central concept of such descriptions is the unity of the background pathophysiologic process and the interaction between the elements. ${ }^{\left({ }^{20}\right)}$ The PS is highly associated with the metabolic syndrome. ${ }^{(5)}$ Furthermore, accumulating evidence supports an association between NAFLD and metabolic syndrome, ${ }^{(21)}$ where NAFLD and metabolic syndrome, being that NAFLD is considered the hepatic manifestation of the metabolic syndrome. ${ }^{(22)}$ Our study did not include none of the components of the syndrome for comparison with the PS and NAFLD.

Our findings demonstrated that fat accumulation in pancreas is correlated to NAFLD. It was also shown that the ultrasound is very resourceful when it comes evaluation of fat grade.

\section{REFERENCES}

1. Ogilvie RF. The islands of Langerhans in 19 cases of obesity. J Pathol Bacteriol. 1933;37:473-8.
2. Smits MM, van Geenen EJ. The clinical significance of pancreatic steatosis. Nat Rev Gastroenterol Hepatol. 2011;8:169-77.

3. Patel S, Bellon EM, Haaga J, Park CH. Fat replacement of the exocrine pancreas. AJR Am J Roentgenol. 1980;135:843-5.

4. Mortelé KJ, Rocha TC, Streeter JL, Taylor AJ. Multimodality imaging of pancreatic and biliary congenital anomalies. Radiographics. 2006;26:715-31.

5. Wu WC, Wang CY. Association between nonalcoholic fatty pancreatic disease (NAFPD) and the metabolic syndrome: case-control retrospective study. Cardiovasc Diabetol. 2013;12:77.

6. Ferolla SM, Armiliato GN, Couto CA, Ferrari TC. Probiotics as a complementary therapeutic approach in nonalcoholic fatty liver disease. World J Hepatol. 2015;7:559-65.

7. Lingvay I, Esser V, Legendre JL, Price AL, Wertz KM, Adams-Huet B, et al. Noninvasive quantification of pancreatic fat in humans. $J$ Clin Endocrinol Metab 2009;94:4070-76.

8. Lee JS, Kim SH, Jun DW, Han JH, Jang EC, Park $J Y$, et al. Clinical implications of fatty pancreas: correlations between fatty pancreas and metabolic syndrome. World J Gastroenterol. 2009;15:1869-75.

9. Yeh WC, Jeng YM, Li CH, Lee PH, Li PC. Liver steatosis classification using high-frequency ultrasound. Ultrasound Med Biol. 2005;31:599605.

10. Olsen TS. Lipomatosis of the pancreas in autopsy material and its relation to age and overweight. Acta Pathol Microbiol Scand. 1978;86A:367-73.

11. Sepe PS, Ohri A, Sanaka S, Berzin TM, Sekhon $S$, Bennett $G$, et al. A prospective evaluation of fatty pancreas by using EUS. Gastrointest Endosc. 2011;73:987-93.

12. Saisho Y, Butler AE, Meier JJ, Monchamp T, Allen-Auerbach M, Rizza RA, et al. Pancreas volumes in humans from birth to age one hundred taking into account sex, obesity, and presence of type-2 diabetes. Clin Anat. 2007;20:933-42. 
13. Sijens PE, Edens MA, Bakker SJ, Stolk RP. MRI-determined fat content of human liver, pancreas and kidney. World J Gastroenterol. 2010;16:1993-8.

14. Gullo L, Salizzoni E, Serra C, Calculli L, Bastagli L, Migliori M. Can pancreatic steatosis explain the finding of pancreatic hyperenzymemia in subjects with dyslipidemia? Pancreas. 2006;33:351-3.

15. Verma AR, Papalois V. Evaluating steatosis in pancreatic transplant. Exp Clin Transplant. 2011;9:159-64.

16. Glaser J, Stienecker K. Pancreas and aging: a study using ultrasonography. Gerontology. 2000;46:93-9.

17. Noronha M, Salgadinho A, Ferreira De Almeida MJ, Dreiling DA, Bordalo O. Alcohol and the pancreas. I. Clinical associations and histopathology of minimal pancreatic inflammation. Am J Gastroenterol. 1981;76:114-9.
18. Gan L, Chitturi S, Farrell GC. Mechanisms and implications of age-related changes in the liver: nonalcoholic Fatty liver disease in the elderly. Curr Gerontol Geriatr Res. 2011;2011:831536.

19. Festi D, Colecchia A, Sacco T, Bondi M, Roda E, Marchesini G. Hepatic steatosis in obese patients: clinical aspects and prognostic significance. Obes Rev. 2004;5:27-42.

2O. Khoshdel AR, Carney SL, Gillies A. Circulatory syndrome: an evolution of the metabolic syndrome concept! Curr Cardiol Rev. 2O12;8:6876.

21. Dietrich P, Hellerbrand C. Non-alcoholic fatty liver disease, obesity and the metabolic syndrome. Best Pract Res Clin Gastroenterol. 2014;28:637-53.

22. Kim CH, Younossi ZM. Nonalcoholic fatty liver disease: a manifestation of the metabolic syndrome. Cleve Clin J Med. 2008;75:721-8. 